\title{
A mathematical model of the rail track presented as a bar on elastic and dissipative supports under the influence of moving loads
}

\author{
Alexander Darenskiy ${ }^{1}$, Volodymyr Vitolberg ${ }^{1}$, Denis Fast $^{1}$, Andrii Klymenko ${ }^{1}$ and \\ Yaroslav Leibuk ${ }^{1}$ \\ ${ }^{1}$ Department of track and track facilities, Ukrainian State University of Railway Transport, Feierbakh \\ Square 7, 61050, Kharkiv, Ukraine
}

\begin{abstract}
At present, the most common track model is the one in which rails are presented as bars of infinite length rested on continuous elastic foundation. However, some specialists consider the model to be rather ideal for railways in terms of track and the technical state of track. Calculation of track as a bar rested on numerous elastic supports with variable characteristics of stiffness under static loads has shown that application of methods of elastic foundation gives results understated by $17-24 \%$. The study presents mathematic models of the vehicle/track dynamic system, and a design scheme of track presented as a bar on numerous elastic dissipative supports with non-linear characteristics, which is taken on the base of this system. The authors developed models and methods to define the reduced vertical stiffness of the track in the wheel/rail contact point, which considers rail elastic and geometric characteristics, stiffness of supports, distance between supports and distributed track mass. The value of stiffness is variable by time for each wheel at any time and various for the vehicle's wheels. The mathematical model proposed has been implemented in Matlab software and will make it possible to conduct numerical research into the track/vehicle dynamics.
\end{abstract}

\section{Introduction}

An investigation into rail track operation under loads from the rolling stock by applying numerical methods makes it possible to obtain information on the impact on track and features of the deflected mode of the whole structure in various operational conditions at much lower costs if compared with conducting experiments. Numerical methods make it possible to specify the input design data, which is virtually impossible in experiments.

However, numerical methods can only be implemented when mathematical models adequately reproduce the actual operation of both the vehicle/track dynamic system and track structure. It refers entirely to simulation of the track/vehicle interaction. Thus, simulation of the track/vehicle interaction is an urgent task.

\footnotetext{
* Corresponding author: ppx xiit@kart.edu.ua
} 


\section{The analysis of studies and publications}

On the basis of theoretical research conducted by E.M. Bromberg, M.F. Verigo, A.Ya. Kogan, G.M. Shahunjanc [1-4] and others mathematical models of the track/rolling stock interaction and the deflected mode of track based on that design scheme were developed. A large number of experiments have revealed that for main line railways the theoretical and experiment findings have very good convergence.

However, some specialists (K.D. Belykh [5], N.S. Nikerov [6] and others) consider this model to be rather ideal for railways in terms of track and the technical state of the track. V.V. Rybkin when calculating track as a bar rested on numerous elastic supports with variable characteristics of stiffness under static loads showed that methods of elastic foundation when applied gave results understated by 17-24\% [7].

The study [8] gives mathematic models of the dynamic vehicle/track system on the base of which a design scheme of track as a bar on numerous elastic dissipative supports with non-linear characteristics was taken. Implementation of these models in Matlab software allows us to define spatial forces from rolling stock on track.

However, these models do not consider the inertial characteristics of track, which limits their application by cases when speeds do not exceed $40 \mathrm{~km} / \mathrm{h}$. The mentioned conditions exist, for example, on non-public railways.

\section{The general statement of the problem}

The study deals with solution to the problem of absence at present a mathematical model of track as a bar rested on numerous elastic and dissipative supports with consideration of the inertial track characteristics. The development of such a model will considerably increase the accuracy of calculation. The objective of the research is development of a mathematical model of track as a bar on elastic supports, which is inertial under moving loads from the rolling stock.

\section{The basic part}

The study [9] presents the differential equation of transverse vibrations of bars under the influence of transverse loads changing by the harmonic law:

$$
y(x)=y_{0} A s x+\frac{y_{0}^{\prime}}{s} B s x-\frac{M_{0}}{s^{2} E I} C s x-\frac{Q_{0}}{s^{3} E I} D s x-\frac{q}{s^{4} E I}\left(A_{s x}-1\right),
$$

where $y_{0}, y_{0}^{\prime}, M_{0}, Q_{0}$ are the initial parameters: bend, turning angle, bending moment, transverse force at the origin of coordinates, respectively; $A s x, B s x, C s x, D s x$ are Krylov functions;

$E$ is the coefficient of elasticity;

$I$ is the moment of inertia of the cross-section;

$s$ is the characteristic number defined by the ratio:

$$
s^{4}=\frac{m \theta^{2}}{E I},
$$

where $m$ is the distributed track mass involved in vibration; $\theta$ is the vibration frequency. 


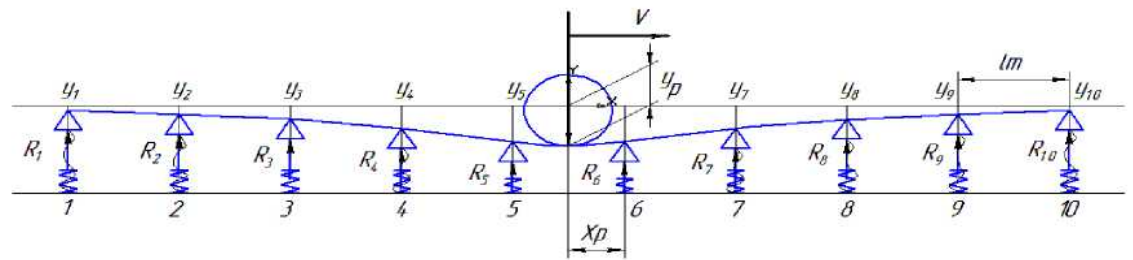

Fig. 1. The design scheme of track on elastic supports under the moving force

Let us consider track as a design scheme presented in Fig.1. A large amount of research, the results of which are presented, for example, in [8], has shown that a bending wave of the rail does not exceed $2.5 \mathrm{~m}$ from the load application point. Therefore, let us consider the track as a bar rested on ten supports. Under the influence of concentrated force moving along the track equation (1) is generally presented as:

$$
\begin{aligned}
& y(x)=\frac{1}{s^{3} E I}\left(-R_{1} D s\left(x-x_{1}\right)-R_{2} D s\left(x-x_{2}\right)-R_{3} D s\left(x-x_{3}\right)-R_{4} D s\left(x-x_{4}\right)-R_{5} D s\left(x-x_{5}\right)-\right. \\
& \left.-R_{6} D s\left(x-x_{6}\right)-R_{7} D s\left(x-x_{7}\right)-R_{8} D s\left(x-x_{8}\right)-R_{9} D s\left(x-x_{9}\right)-R_{10} D s\left(x-x_{10}\right)+P D s\left(x-x_{P}\right)\right),
\end{aligned}
$$

where $R_{i}$ is the reaction of the supports;

$P$ is the wheel load;

$x_{i}$ is the distance from each support to the cross-section under consideration.

$$
\begin{aligned}
& y(1)=\frac{1}{s^{3} E I}\left(-R_{1} D s(l-l)-R_{2} D s(l-2 l)-R_{3} D s\left(l-3 l_{u u}\right)-R_{4} D s(l-4 l)-R_{5} D s(l-5 l)-\right. \\
& \left.-R_{6} D s(l-6 l)-R_{7} D s(l-7 l)-R_{8} D s(l-8 l)-R_{9} D s(l-9 l)--R_{10} D s(l-10 l)+P D s\left(l-x_{P}\right)\right) \\
& y(2)=\frac{1}{s^{3} E I}\left(-R_{1} D s(2 l-l)-R_{2} D s(2 l-2 l)-R_{3} D s(2 l-3 l)-R_{4} D s(2 l-4 l)-R_{5} D s(2 l-5 l)-\right. \\
& \left.-R_{6} D s(2 l-6 l)-R_{7} D s(2 l-7 l)-R_{8} D s(2 l-8 l)-R_{9} D s(2 l-9 l)-R_{10} D s(2 l-10 l)+P D s\left(2 l-x_{P}\right)\right) \\
& y(3)=\frac{1}{s^{3} E I}\left(-R_{1} D s(3 l-l)-R_{2} D s(3 l-2 l)-R_{3} D s(3 l-3 l)-R_{4} D s(3 l-4 l)-R_{5} D s(3 l-5 l)-\right. \\
& \left.-R_{6} D s(3 l-6 l)-R_{7} D s(3 l-7 l)-R_{8} D s(3 l-8 l)-R_{9} D s(3 l-9 l)-R_{10} D s(3 l-10 l)+P D s\left(3 l-x_{P}\right)\right) \\
& y(4)=\frac{1}{s^{3} E I}\left(-R_{1} D s(4 l-l)-R_{2} D s(4 l-2 l)-R_{3} D s(4 l-3 l)-R_{4} D s(4 l-4 l)-R_{5} D s(4 l-5 l)-\right. \\
& \left.-R_{6} D s(4 l-6 l)-R_{7} D s(4 l-7 l)-R_{8} D s(4 l-8 l)-R_{9} D s(4 l-9 l)-R_{10} D s(4 l-10 l)+P D s\left(4 l-x_{P}\right)\right) \\
& y(5)=\frac{1}{s^{3} E I}\left(-R_{1} D s(5 l-l)-R_{2} D s(5 l-2 l)-R_{3} D s(5 l-3 l)-R_{4} D s(5 l-4 l)-R_{5} D s(5 l-5 l)-\right. \\
& \left.-R_{6} D s(5 l-6 l)-R_{7} D s(5 l-7 l)-R_{8} D s(5 l-8 l)-R_{9} D s(5 l-9 l)-R_{10} D s(5 l-10 l)+P D s\left(5 l-x_{P}\right)\right) \\
& y(6)=\frac{1}{s^{3} E I}\left(-R_{1} D s(6 l-l)-R_{2} D s(6 l-2 l)-R_{3} D s(6 l-3 l)-R_{4} D s(6 l-4 l)-R_{5} D s(6 l-5 l)-\right. \\
& \left.-R_{6} D s(6 l-6 l)-R_{7} D s(6 l-7 l)-R_{8} D s(6 l-8 l)-R_{9} D s(6 l-9 l)-R_{10} D s(6 l-10 l)+P D s\left(6 l-x_{P}\right)\right) \\
& y(7)=\frac{1}{s^{3} E I}\left(-R_{1} D s(7 l-l)-R_{2} D s(7 l-2 l)-R_{3} D s(7 l-3 l)-R_{4} D s(7 l-4 l)-R_{5} D s(7 l-5 l)-\right. \\
& -R_{6} D s(7 l-6 l)-R_{7} D s(7 l-7 l)-R_{8} D s(7 l-8 l)-R_{9} D s(7 l-9 l)-R_{10} D s(7 l-10 l)+P D s\left(7 l-x_{P}\right) \\
& y(8)=\frac{1}{s^{3} E I}\left(-R_{1} D s(8 l-l)-R_{2} D s(8 l-2 l)-R_{3} D s(8 l-3 l)-R_{4} D s(8 l-4 l)-R_{5} D s(8 l-5 l)-\right. \\
& -R_{6} D s(8 l-6 l)-R_{7} D s(8 l-7 l)-R_{8} D s(8 l-8 l)-R_{9} D s(8 l-9 l)-R_{10} D s(8 l-10 l)+P D s\left(8 l-x_{P}\right) \\
& y(9)=\frac{1}{s^{3} E I}\left(-R_{1} D s(9 l-l)-R_{2} D s(9 l-2 l)-R_{3} D s(9 l-3 l)-R_{4} D s(9 l-4 l)-R_{5} D s(9 l-5 l)-\right. \\
& \left.-R_{6} D s(9 l-6 l)-R_{7} D s(9 l-7 l)-R_{8} D s(9 l-8 l)-R_{9} D s(9 l-9 l)-R_{10} D s(9 l-10 l)+P D s\left(9 l-x_{P}\right)\right) \\
& y(10)=\frac{1}{s^{3} E I}\left(-R_{1} D s(10 l-l)-R_{2} D s(10 l-2 l)-R_{3} D s(10 l-3 l)-R_{4} D s(10 l-4 l)-R_{5} D s(10 l-5 l)-\right. \\
& -R_{6} D s(10 l-6 l)-R_{7} D s(10 l-7 l)-R_{8} D s(10 l-8 l)-R_{9} D s(10 l-9 l)-R_{10} D s(10 l-10 l)+P D s\left(10 l-x_{P}\right)
\end{aligned}
$$


By using the basic equation (3), equations were compiled for each of the ten supports under the influence of the force $P$.

In these equations, the distance from the nearest support to the load application point is defined as:

$$
x_{P}=5 l+\left(1-\left\{\frac{x_{n i}}{l}\right\}\right) \cdot l,
$$

where $\left\{\frac{x_{n i}}{l}\right\}$ is the fractional part of the ratio $\frac{x_{n i}}{l}$;

System (4) can be presented as a matrix:

$$
A \cdot y=H
$$

where A is the matrix of elastic and inertial characters of the track; $y$ is the matrix of unknown deformations in the supports;

$H$ is the external load matrix;

The matrix of elastic and inertial characteristics of the track is of the form.

$$
\left\{\begin{array}{cccccc}
s^{3} E I & c D(l-2 l) & c D(l-3 l) & c D(l-4 l) & c D(l-5 l) & \\
c D(2 l-l) & s^{3} E I & c D(2 l-3 l) & c D(2 l-4 l) & c D(2 l-5 l) & \\
c D(3 l-l) & c D(3 l-2 l) & s^{3} E I & c D(3 l-4 l) & c D(3 l-5 l) & \\
c D(4 l-l) & c D(4 l-2 l) & c D(4 l-3 l) & s^{3} E I & c D(4 l-5 l) & \\
c D(5 l-l) & c D(5 l-2 l) & c D(5 l-3 l) & c D(5 l-4 l) & s^{3} E I & \\
c D(6 l-l) & c D(6 l-2 l) & c D(6 l-3 l) & c D(6 l-4 l) & c D(6 l-5 l) & \\
c D(7 l-l) & c D(7 l-2 l) & c D(7 l-3 l) & c D(7 l-4 l) & c D(7 l-5 l) & \\
c D(8 l-l) & c D(8 l-2 l) & c D(8 l-3 l) & c D(8 l-4 l) & c D(8 l-5 l) & \\
c D(9 l-l) & c D(9 l-2 l) & c D(9 l-3 l) & c D(9 l-4 l) & c D(9 l-5 l) & \\
c D(10 l-l) & c D(10 l-2 l) & c D(10 l-3 l) & c D(10 l-4 l) & c D(10 l-5 l) & \\
& & & & & \\
& c D(l-6 l) & c D(l-7 l) & c D(l-8 l) & c D(l-9 l) & c D(l-10 l) \\
& c D(2 l-6 l) & c D(2 l-7 l) & c D(2 l-8 l) & c D(2 l-9 l) & c D(2 l-10 l) \\
& c D(3 l-6 l) & c D(3 l-7 l) & c D(3 l-8 l) & c D(3 l-9 l) & c D(3 l-10 l) \\
& c D(4 l-6 l) & c D(4 l-7 l) & c D(4 l-8 l) & c D(4 l-9 l) & c D(4 l-10 l) \\
& c D(5 l-6 l) & c D(5 l-7 l) & c D(5 l-8 l) & c D(5 l-9 l) & c D(5 l-10 l) \\
& s^{3} E I & c D(6 l-7 l) & c D(6 l-8 l) & c D(6 l-9 l) & c D(6 l-10 l) \\
& c D(7 l-6 l) & s^{3} E I & c D(7 l-8 l) & c D(7 l-9 l) & c D(7 l-10 l) \\
& c D(8 l-6 l) & c D(8 l-7 l) & s^{3} E I & c D(8 l-9 l) & c D(8 l-10 l) \\
& c D(9 l-6 l) & c D(9 l-7 l) & c D(9 l-8 l) & s^{3} E I & c D(9 l-10 l) \\
& c D(10 l-6 l) & c D(10 l-7 l) & c D(10 l-8 l) & c D(10 l-9 l) & s^{3} E l
\end{array}\right\}
$$

The other matrices are of the form: 


$$
y=\left\{\begin{array}{l}
y_{1} \\
y_{2} \\
y_{3} \\
y_{4} \\
y_{5} \\
y_{6} \\
y_{7} \\
y_{8} \\
y_{9} \\
y_{10}
\end{array}\right\},(8) \quad H=\left\{\begin{array}{l}
P(t) D s\left(l-x_{P}\right) \\
P(t) D s\left(2 l-x_{p}\right) \\
P(t) D s\left(3 l-x_{p}\right) \\
P(t) D s\left(4 l-x_{p}\right) \\
P(t) D s\left(5 l-x_{p}\right) \\
P(t) D s\left(6 l-x_{p}\right) \\
P(t) D s\left(7 l-x_{p}\right) \\
P(t) D s\left(8 l-x_{p}\right) \\
P(t) D s\left(9 l-x_{P}\right) \\
P(t) D s\left(10 l-x_{P}\right)
\end{array}\right\},(9)\left\{R_{i}\right\}=\left\{\begin{array}{l}
R_{1}=y_{1} \cdot c \\
R_{2}=y_{2} \cdot c \\
R_{3}=y_{3} \cdot c \\
R_{4}=y_{4} \cdot c \\
R_{5}=y_{5} \cdot c \\
R_{6}=y_{6} \cdot c \\
R_{7}=y_{7} \cdot c \\
R_{8}=y_{8} \cdot c \\
R_{9}=y_{9} \cdot c \\
R_{10}=y_{10} \cdot c
\end{array}\right\} .
$$

The number of unknown support bends in system (4) is equal to the number of equations; therefore matrix A of equation (6) is square with ten lines and columns. Let us indicate as $\Delta$ the determinant of matrix A (7), as $\Delta_{j}$ the determinant, which is obtained from $\Delta$ by deleting the $\mathrm{j}^{\text {th }}$ column $(j=1, j=2 \ldots j=10)$ and insert instead the column of absolute terms $b_{j}$ of matrix $H(9)$. By Cramer's rule, the elements $y_{j}$ of matrix (8) can be defined as:

$$
y_{j}=\frac{\Delta_{j}}{\Delta} .
$$

This equation, as well as expressions (7), (8) and (9), allows calculating elastic depressions of supports and reactions in them (matrix 10).

The vertical stiffness of track in the wheel/rail contact point can be defined on the basis of the following considerations. By taking the external load as equal to 1 , let us define elastic depressions of all supports $R_{i}$ under the unit force of equation (11).

The value of rail depression in the wheel/rail contact point can be defined as:

$$
y_{e}=y_{5} \frac{x_{p}}{l}+y_{6} \frac{\left(l-x_{p}\right)}{l}+\frac{\left(l-x_{p}\right)^{2} x_{p}^{2}}{3 E I l} .
$$

So the stiffness required is equal to:

$$
C_{b}=\frac{1}{y_{e}} .
$$

\section{Conclusions}

The authors propose the methods to define the reduced vertical stiffness of track in the wheel/rail contact point, which allows consideration of elastic and geometric rail characteristics, stiffness of supports, distance between supports and distributed track mass. The stiffness value is variable by time for each wheel at any time and various for the vehicle's wheels. The mathematical model proposed was applied in Matlab software. 


\section{References}

1. V.G. Al'breht, E.M. Bromberg, N.B. Zverev, Besstykovoj put', 206 (1982).

2. E.M. Bromberg, M.F. Verigo, V.N. Danilov, Vzaimodejstvie puti i podvizhnogo sostava, 280 (1956).

3. G.M. Shahunjants, Zheleznodorozhnyj put', Monografija, 497 (1987).

4. M.F. Verigo, A.J. Kogan, Vzaimodejstvie puti i podvizhnogo sostava, 589 (1986).

5. K.D. Belyh, M.K. Umanov, G.N. Malyshko, Metallurgicheskaja promyshlennost', 5, 7879 (1976).

6. N.S. Nisirov, Trudy LIIZhT, 416, 26-35 (1977).

7. V.I. Klimov, V.V. Rybkin, Trudy DIITa, 32(244), 72-84 (1987).

8. O.M. Darens'kij, Teoretichni ta eksperimental'ni doslizhennja roboti zaliznichnih kolij promislovogo transportu. Kharkiv, 204 (2011).

9. O.M Darens'kij, Y.S. Leibuk, Information and control systems at railway transport. (to be published).

10. K.Wang, C.Huang, W. Zhai, P. Liu, S. Wang, Journal of Traffic and Transportation Engineering (English Edition), 1, 209-220 (2014).

11. S. Dailydka, Transport, (3)23, 236-239 (2008).

12. J. Otero, Journal of Rail and Rapid Transit, 1(226), 62-71 (2011). 